\title{
Direct visualization of the complete evolution of femtosecond laser-induced surface structural dynamics of metals
}

\begin{abstract}
Ranran Fang ${ }^{1,2}$, Anatoliy Vorobyev ${ }^{1}$ and Chunlei Guo ${ }^{1,3}$
Despite extensive studies of femtosecond laser-material interactions, even the simplest morphological responses following femtosecond pulse irradiation have not been fully resolved. Past studies have revealed only partial dynamics. Here we develop a zerobackground and high-contrast scattered-light-based optical imaging technique through which we capture, for the first time, the complete temporal and spatial evolution of the femtosecond laser-induced morphological surface structural dynamics of metals from start to finish, that is, from the initial transient surface fluctuations, through melting and ablation, to the end of resolidification. We find that transient surface structures first appear at a delay time on the order of $100 \mathrm{ps}$, which is attributed to ablation driven by pressure relaxation in the surface layer. The formation dynamics of the surface structures at different length scales are individually resolved, and the sequence of their appearance changes with laser fluence is found. Cooling and complete resolidification, observed here for the first time, are shown to occur more slowly than previously predicted by two orders of magnitude. We examine and identify the mechanisms driving each of these dynamic steps. The visualization and control of morphological surface structural dynamics not only are of fundamental importance for understanding femtosecond laser-induced material responses but also pave the way for the design of new material functionalities through surface structuring.
\end{abstract}

Light: Science \& Applications (2017) 6, e16256; doi:10.1038/lsa.2016.256; published online 10 March 2017

Keywords: ablation; femtosecond laser; surface nano/microstructures; ultrafast dynamics; ultrafast imaging

\section{INTRODUCTION}

Femtosecond (fs) laser-material interactions have been extensively studied in the past ${ }^{1-5}$. However, a clear understanding of even the simplest morphological responses is still lacking; direct visualization of the complete ultrafast structural dynamics in metals has never been achieved. Interestingly, fs laser-induced morphological changes often drastically alter a material's physical properties. For example, surface nano- and microstructuring induced by fs laser pulses has led to the creation of various materials with novel effects, such as black silicon, black and colored metals, and superhydrophilic, superhydrophobic, and multifunctional surfaces ${ }^{2,3}$. These highly functionalized surfaces have found a wide range of applications throughout the physical and biological sciences ${ }^{1-5}$.

Ultrafast morphological and crystalline structural dynamics have been studied using various techniques, including X-ray diffraction ${ }^{6,7}$, electron diffraction ${ }^{8}$, diffractive XUV imaging, and optical imaging $^{10-16}$. Ultrafast X-ray and electron diffraction studies are more suitable for investigating long-range disorder phenomena in crystalline materials, such as melting ${ }^{8}$. In a more recent work on X-ray diffraction combined with photon-correlation spectroscopy, real-space images were extracted through phase retrieval at delay times of up to $15 \mathrm{ps}$, but such retrieval becomes increasingly more difficult and less reliable at longer time delays ${ }^{17}$. Diffractive imaging using time-resolved coherent XUV scattering in a transmission geometry can also be applied for indirect imaging, in which a real image is reconstructed from a diffraction pattern ${ }^{9}$. Optical techniques are more suitable for real-space imaging. However, optical imaging is usually achieved by collecting specular reflections ${ }^{10-16}$ and is more suitable for resolving relatively large changes in reflection, such as Newton-ring formation ${ }^{11-15}$. The strong background of specular reflections makes it difficult to discern the small changes in reflection that are often associated with nano/microstructure formation. Here we develop an ultrafast optical imaging technique to study the structural dynamics of metals by utilizing scattered light instead of specular reflections. Scattered-light imaging has been used to study biological and organic materials because scattering is a prevalent form of light interaction with inhomogeneous media, but scattered-light imaging has not been explored for studying the structural dynamics of solid surfaces ${ }^{18}$. The scattered-light imaging provides a near-zero background and high contrast, allowing us to obtain high-quality real-space images of the structural dynamics of metals and resolve, for the first time, the complete temporal and spatial evolution of fs laser-induced morphological surface structure formation from start to finish in metals.

${ }^{1}$ The Institute of Optics, University of Rochester, Rochester, NY 14627, USA; ${ }^{2}$ Present address: School of Science, Chongqing University of Posts and Telecommunications, Chongqing, China and ${ }^{3}$ The Guo China-US Photonics Laboratory, Changchun Institute of Optics, Fine Mechanics and Physics, Chinese Academy of Sciences, Changchun, China Correspondence: C Guo, Email: guo@optics.rochester.edu

Received 9 September 2016; revised 17 November 2016; accepted 21 November 2016; accepted article preview online 23 November 2016 


\section{MATERIALS AND METHODS}

Ultrafast pump-probe imaging

Our ultrafast pump-probe imaging setup is depicted in Figure 1a. We use an amplified Ti:sapphire laser system that generates $65-\mathrm{fs}$ pulses with a pulse energy of $1 \mathrm{~mJ}$, a repetition rate of $1 \mathrm{kHz}$, and a central wavelength of $800 \mathrm{~nm}$. A fast electromechanical shutter is used to select a single pulse. A pump beam induces the formation of surface structures on $\mathrm{Zn}$. Surface nano/microstructures are produced by a single laser pulse at a fluence in the range of $0.1-1.0 \mathrm{~J} \mathrm{~cm}^{-2}$, under which extensive nano/microstructure formation is observed ${ }^{19}$. The incidence angle of the pump beam is $36^{\circ}$. The $\mathrm{Zn}$ sample is first mechanically polished using $0.05-\mu \mathrm{m} \mathrm{Al}_{2} \mathrm{O}_{3}$ powder, washed in methanol, and finally cleaned using Ar plasma. The probe beam is used to provide stroboscopic illumination of the surface structures induced by the pump beam. An optical delay line is used to provide various time delays, ranging from 0 to $408 \mathrm{~ns}$, between the pump and probe pulses. A delay of $408 \mathrm{~ns}$ is achieved by allowing the beam to propagate over $120 \mathrm{~m}$ through multiple reflections. A zero delay is achieved by using the autocorrelation technique using a BBO crystal.
After passing the delay line, the probe pulse is directed onto the $\mathrm{BBO}$ crystal to generate second harmonics at $400 \mathrm{~nm}$. A blue band-pass filter is used to block the fundamental light after the BBO crystal. The $400-\mathrm{nm}$ probe pulse is incident on the sample at an angle of $18^{\circ}$. A 50 - $\mathrm{mm}$ (numerical aperture $=0.25$ ) long-working-distance objective is used to image the sample surface onto the sensor of a CCD camera $(1600 \times 1200$ pixels). The CCD camera is positioned normal to the sample surface. A 400-nm narrow band-pass filter is placed in front of the imaging objective to block the pump light. The imaging optics we use are capable of resolving both nanoclusters (microscale size) and microstructures. At a fixed laser fluence, we acquire a sequence of three surface images. The first snapshot is taken $10 \mathrm{~s}$ before the pump laser pulse irradiation for the identification of initial structures, if any, that may be present on the surface. The second snapshot of the surface is taken at a preset time delay. The third snapshot is taken $10 \mathrm{~s}$ after the pump laser pulse irradiation to record the permanent surface structures produced by the laser pulse. By recording such sequences of surface images at different time delays and laser fluences, we can track the formation dynamics of the transient surface structures at different
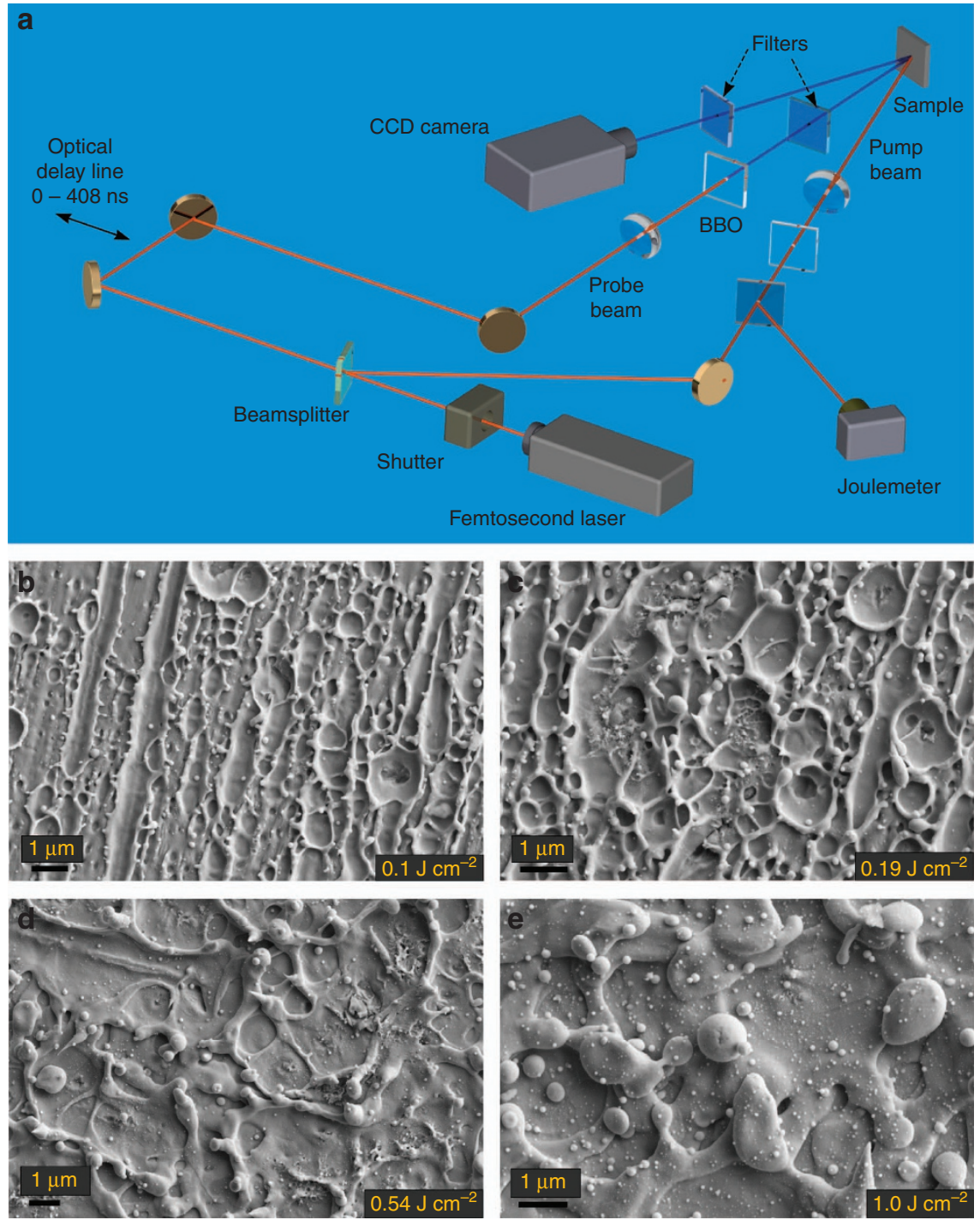

Figure 1 (a) Ultrafast pump-probe imaging setup. (b-e) SEM images of surface nano/microstructures of $\mathrm{Zn}$ in the center of the irradiated spot at various laser fluences, as indicated in the figure. 
delays and identify the effect of the laser fluence on their evolution, including transient surface structural fluctuations and solidification. The scattered-light images may contain speckle, but the speckle is not pronounced for femtosecond pulse imaging because femtosecond pulses have a very short coherence length.

\section{Reflectance measurements}

The optical reflection from a surface will change when the surface is irradiated with a high-intensity laser beam, and the surface reflectance at a given fluence is crucial for our study. Therefore, we also measure the surface reflectance at different fluences by using a hemiellipsoidal reflector to collect both the specular and diffuse light reflection ${ }^{20}$. The studied sample is placed at the internal focal point of the reflector. The reflected light is collected at the external focal point of the reflector, and its energy $E_{\mathrm{R}}$ is measured using a joulemeter. The sample is tilted at the same incidence angle as in our imaging setup with respect to the pump beam $\left(36^{\circ}\right)$. A second joulemeter is used to measure the incident pulse energy, $E_{\mathrm{I}}$. The reflectance $R=E_{\mathrm{R}} / E_{\mathrm{I}}$ is obtained from the measured $E_{\mathrm{R}}$ and $E_{\mathrm{I}}$. To calibrate our reflectance measurements, we compare the reflectance under low-intensity laser light (below the damage threshold) with that measured at $800 \mathrm{~nm}$ using a Perkin-Elmer Lambda 900 spectrophotometer with an integrating sphere.

\section{Characterization of surface structures}

A scanning electron microscope (SEM) and a three-dimensional laser scanning microscope are used to examine the laser-induced surface nano/microstructures after the resolidification of the surface.

\section{RESULTS AND DISCUSSION}

The single-pulse damage threshold of our zinc sample was measured to be $22 \mathrm{~mJ} \mathrm{~cm}^{-2}$. This threshold was determined based on the first appearance of surface modification observed under an optical microscope. Figure 1b-1e are SEM images of some typical surface nano/ microstructures produced in the center of the ablated spot at various laser fluences, where one can see that nanostructures dominate at lower fluences, whereas microstructures dominate at higher fluences. Figure 2 shows the transient structures of $\mathrm{Zn}$ at different delays for a laser fluence of $0.1 \mathrm{~J} \mathrm{~cm}^{-2}$, which is approximately four times larger than the damage threshold. For each delay, the final image of the same spot is also shown for comparison. It is seen that surface structures begin to appear at a time delay of $400 \mathrm{ps}$ (Figure 2c), but a comparison with the structures observed a long time after the laser pulse $(t=\infty)$ (Figure 2d) reveals that the two images show nearly no resemblance, indicating that the material in the irradiated spot is not yet solidified, and therefore, the surface structures observed at this delay are transient. A comparison of Figure 2e and $2 \mathrm{f}$ reveals some similarity at the periphery of the irradiated spot, which indicates that the transient structures begin to solidify at 527 ps and that interestingly, solidification first occurs at the periphery. With increasing delay time, the solidified area increases. Figure $2 \mathrm{~g}$ and $2 \mathrm{~h}$ shows that $\sim 90 \%$ of the surface structures are frozen at $t=50 \mathrm{~ns}$. Finally, Figure $2 \mathrm{i}$ and $2 \mathrm{j}$ shows that the structures become identical to their final configuration at $124 \mathrm{~ns}$, indicating that the surface structures are completely solidified at this time. Figure $1 \mathrm{~b}$ shows that for a fluence of $0.1 \mathrm{~J} \mathrm{~cm}^{-2}$, the surface structures are dominated by extensive nanostructures with dimensions between 30 and $500 \mathrm{~nm}$, and some microstructures. Note that the optical imaging system used here, operating with $400 \mathrm{~nm}$ light, resolves nanoclusters at the micron scale but not individual nanostructures. To fully characterize the surface structural evolution dynamics, three characteristic times are important: (1) the time at
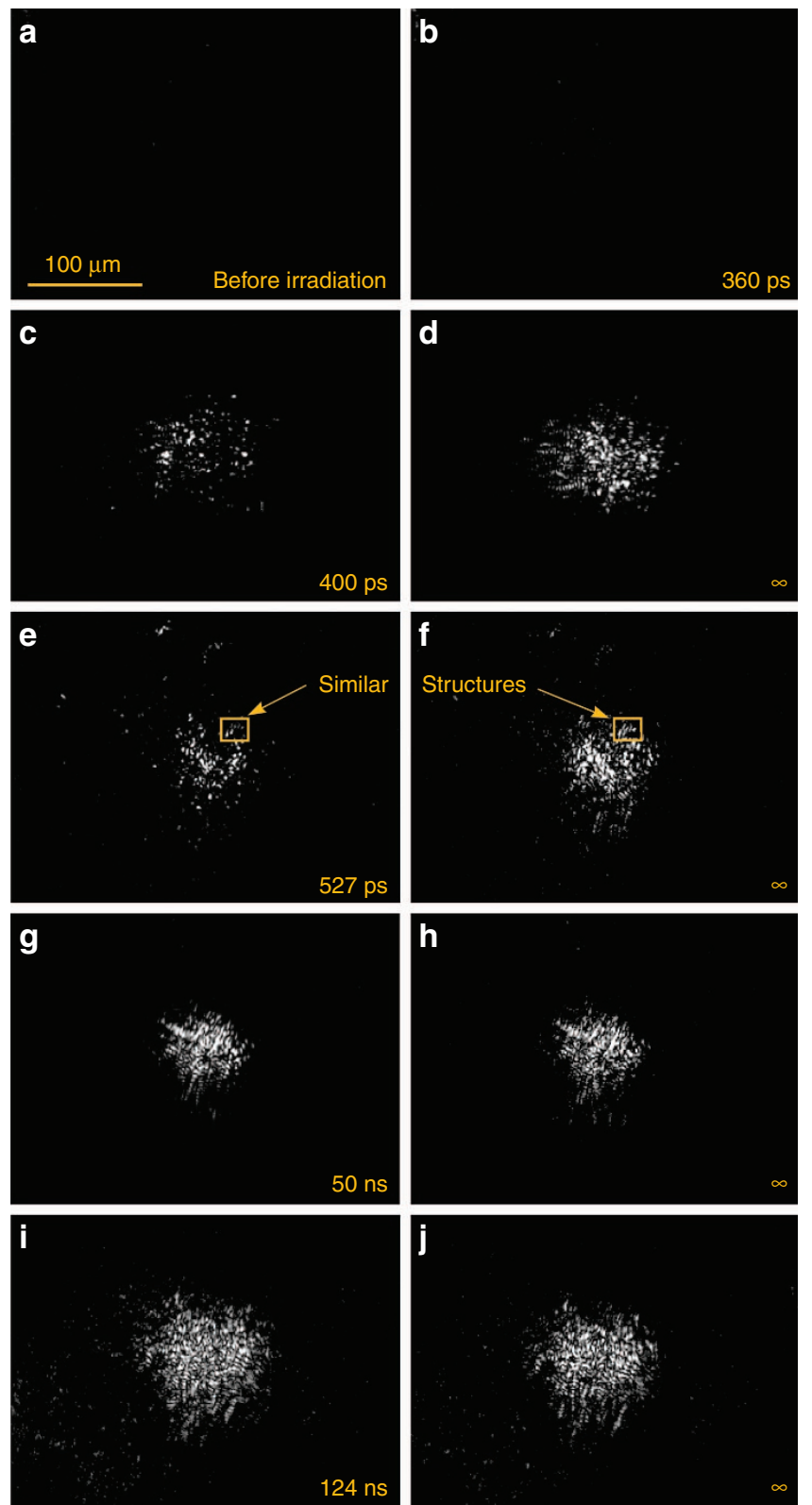

Figure $2(\mathbf{a}-\mathbf{j})$ A comparison of transient surface structures observed at various delay times and final solidified structures following a pump pulse at a fluence of $0.10 \mathrm{~J} \mathrm{~cm}^{-2}$.

which transient surface structures first appear, $t_{1} ;(2)$ the time at which solidification begins, $t_{2}$; and (3) the time at which the surface structures become completely solidified, $t_{3}$. These three characteristic times as found in our study for different pump fluences are summarized in Table 1. Previously, fs laser-excited surface structure formation has been studied for semiconductors ${ }^{12,13}$; in these studies, the time required for complete solidification in silicon was found to be $\sim 75 \mathrm{~ns}$, much shorter than what we observe here for $\mathrm{Zn}$.

First, we study the mechanism of the transient surface structure formation and its associated start time, $t_{1}$. Previous studies have shown that the characteristic time for surface melting following the ultrafast laser irradiation of $\mathrm{Zn}$ is $\sim 3$ ps at laser fluences of $0.1-0.15 \mathrm{~J} \mathrm{~cm}^{-2}$ (Ref. 21). However, our data show that the onset of transient surface structure formation occurs at $\sim 400$ and $\sim 300$ ps at laser fluences of 
0.1 and $0.14 \mathrm{~J} \mathrm{~cm}^{-2}$, respectively. This time difference suggests that the irradiated surface does not undergo a morphological change immediately after melting; instead, there is a time delay between the melting process and the hydrodynamic motion of the melted material that results in the transient morphological fluctuations observed on the surface. In general, transient structural fluctuations can be induced by the flow of the melted material due to a temperature gradient (the Marangoni effect) and the flow due to laser-induced stresses in the melted surface layer. To identify whether the Marangoni effect plays the key role here, we estimate the characteristic timescale $t_{\mathrm{M}}$ of the Marangoni effect as follows ${ }^{22}$ :

$$
t_{\mathrm{M}} \approx \frac{\eta L^{2}}{\left|\gamma_{\mathrm{T}}\right| T_{\mathrm{m}} h}
$$

where $\eta$ is the dynamic viscosity of the melt, $L$ is the typical radial dimension, $h$ is the average melt depth, $T_{\mathrm{m}}$ is the melting point of the metal, and $\left|\gamma_{\mathrm{T}}\right|=|\mathrm{d} \gamma / \mathrm{d} T|$ is the absolute value of the temperature coefficient of the surface tension $\gamma$. Using values of $\eta=2.5 \times 10^{-3} \mathrm{~Pa} \cdot \mathrm{s}$ (Ref. 23), $\left|\gamma_{\mathrm{T}}\right|=0.25 \times 10^{-3} \mathrm{~N} \mathrm{~m}^{-1} \mathrm{~K}^{-1}$ (Ref. 24), $T_{\mathrm{m}}=693 \mathrm{~K}$, and $L=200 \mathrm{~nm}$ (determined from Figure 1b) and assuming $h=30 \mathrm{~nm}$, we obtain $t_{\mathrm{M}} \approx 20 \mathrm{~ns}$, which is two orders of magnitude larger than the observed values of $t_{1}$ (which are on the ps timescale). Therefore, the Marangoni effect does not appear to be the primary cause of the onset of the transient surface structures. However, this does not exclude the Marangoni effect as a factor affecting the formation of surface structures at longer timescales.

Following high-intensity ultrashort laser pulse heating, a highly pressurized melted surface layer can form as a result of isochoric heating, as has been theoretically predicted ${ }^{25,26}$. Pressure relaxation in this melted layer will induce hydrodynamic motion, resulting in morphological fluctuations on the surface. The timescale of these morphological fluctuations is governed by the speed of sound in the melted material ${ }^{26}$ and can be estimated as $t_{\mathrm{p}} \approx L / v_{\mathrm{sl}}$, where $v_{\mathrm{sl}}$ is the speed of sound in liquid $\mathrm{Zn}$. The speed of sound in solid $\mathrm{Zn}$ is $3.8 \times 10^{3} \mathrm{~m} \mathrm{~s}^{-1}$ (Ref. 27). Typically, the speed of sound in liquid metals is 1.5 times smaller than that in solids. Therefore, assuming $v_{\mathrm{sl}}=2.3 \times 10^{3} \mathrm{~m} \mathrm{~s}^{-1}$ and again using the typical radial dimension $L=200 \mathrm{~nm}$ found from Figure $1 \mathrm{~b}$, we obtain $t_{\mathrm{P}} \approx 90 \mathrm{ps}$, which is on the same timescale as our measured $t_{1}$ values. In general, melting occurs at laser fluences near the damage threshold, and ablation occurs at higher fluences. The laser fluence we use in our study is more than four times greater than the damage threshold. Therefore, we believe that the start time of transient surface structure formation, $t_{1}$, as observed in our experiment is associated with ablation driven by pressure relaxation in the surface layer. Figure 3 presents $t_{1}$ and $t_{2}$ as functions of the laser fluence. We can see that $t_{1}$ decreases with laser fluence, which indicates an earlier onset of ablation due to higher pressure in the surface layer.

Figure 4 shows a set of time-resolved surface images acquired at various delays following pump pulse irradiation at a higher fluence of $1.0 \mathrm{~J} \mathrm{~cm}^{-2}$. Somewhat counter- intuitively, we can see that transient surface structures first appear at the edge of the irradiated spot and form a ring, in contrast to what occurs under ablation at lower laser fluences, for which the transient structures first appear in the center (Figure 2c). Over time, the transient surface structures move toward the center. At 5-9.3 ns, the center of the irradiated spot is covered with structures. The solidification of the transient surface structures begins at $\sim 124 \mathrm{~ns}$, as indicated by the resemblance to the final image. At the longest delay investigated in our experiments (408 ns), solidification is not fully complete, as a difference still exists between Figure $4 \mathrm{k}$ and $4 \mathrm{l}$. A comparison of Figures 2 and 4 shows that the spatial sequencing of the transient structures before solidification varies with the laser fluence. To better understand this dependence, we consider surface images acquired at a fixed delay time of 527 ps but at different laser fluences, as shown in Figure 5. We can see that a transition from first emergence of the transient structures in the center to first emergence at the edge occurs at $0.54 \mathrm{~J} \mathrm{~cm}^{-2}$. The SEM images presented in Figure 1 show that at this laser fluence, the surface structures in the

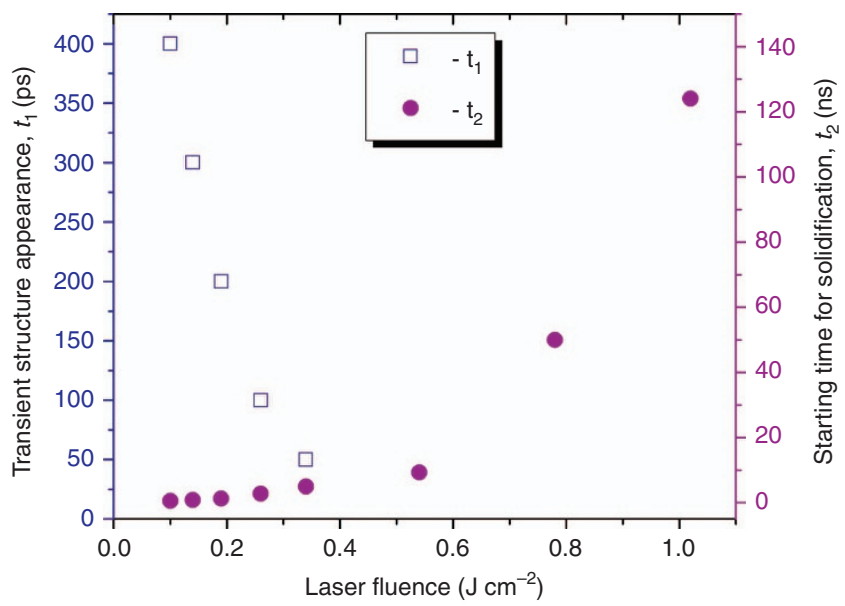

Figure 3 Time at which transient surface structures appear, $t_{1}$, and start time of solidification, $t_{2}$, as functions of laser fluence.

Table 1 Measurements and estimates regarding surface structural dynamics

\begin{tabular}{|c|c|c|c|c|c|c|c|}
\hline$F\left(\mathrm{~J} \mathrm{~cm}^{-2}\right)$ & $t_{1}(\mathrm{ps})$ & $t_{2}(\mathrm{~ns})$ & $t_{3}(\mathrm{~ns})$ & $R$ & $T_{\mathrm{m}}(\mathrm{K})$ & $t_{\text {cool }}(\mathrm{ns})$ & Ablation mechanism \\
\hline 0.022 & - & - & - & 0.57 & 890 & - & \multirow{5}{*}{$\begin{array}{l}\text { Damage threshold } \\
\text { Spallation }\end{array}$} \\
\hline 0.10 & 400 & 0.527 & 123.8 & 0.57 & 2970 & 32 & \\
\hline 0.14 & 300 & 0.86 & 322 & 0.57 & 4080 & 41 & \\
\hline 0.19 & 200 & 1.3 & - & 0.57 & 5430 & 50 & \\
\hline 0.26 & 100 & 2.8 & - & 0.56 & 7320 & 62 & \\
\hline 0.34 & 50 & 5 & - & 0.57 & 9480 & 74 & \multirow[t]{4}{*}{ Phase explosion } \\
\hline 0.54 & - & 9.3 & - & 0.57 & 14880 & 101 & \\
\hline 0.78 & - & 50 & - & 0.55 & 22330 & 130 & \\
\hline 1.0 & - & 124 & - & 0.56 & 28580 & 156 & \\
\hline 1.1 & - & - & - & 0.5 & - & - & \multirow{2}{*}{ Solid-density plasma regime } \\
\hline 1.8 & - & - & - & 0.33 & - & - & \\
\hline
\end{tabular}



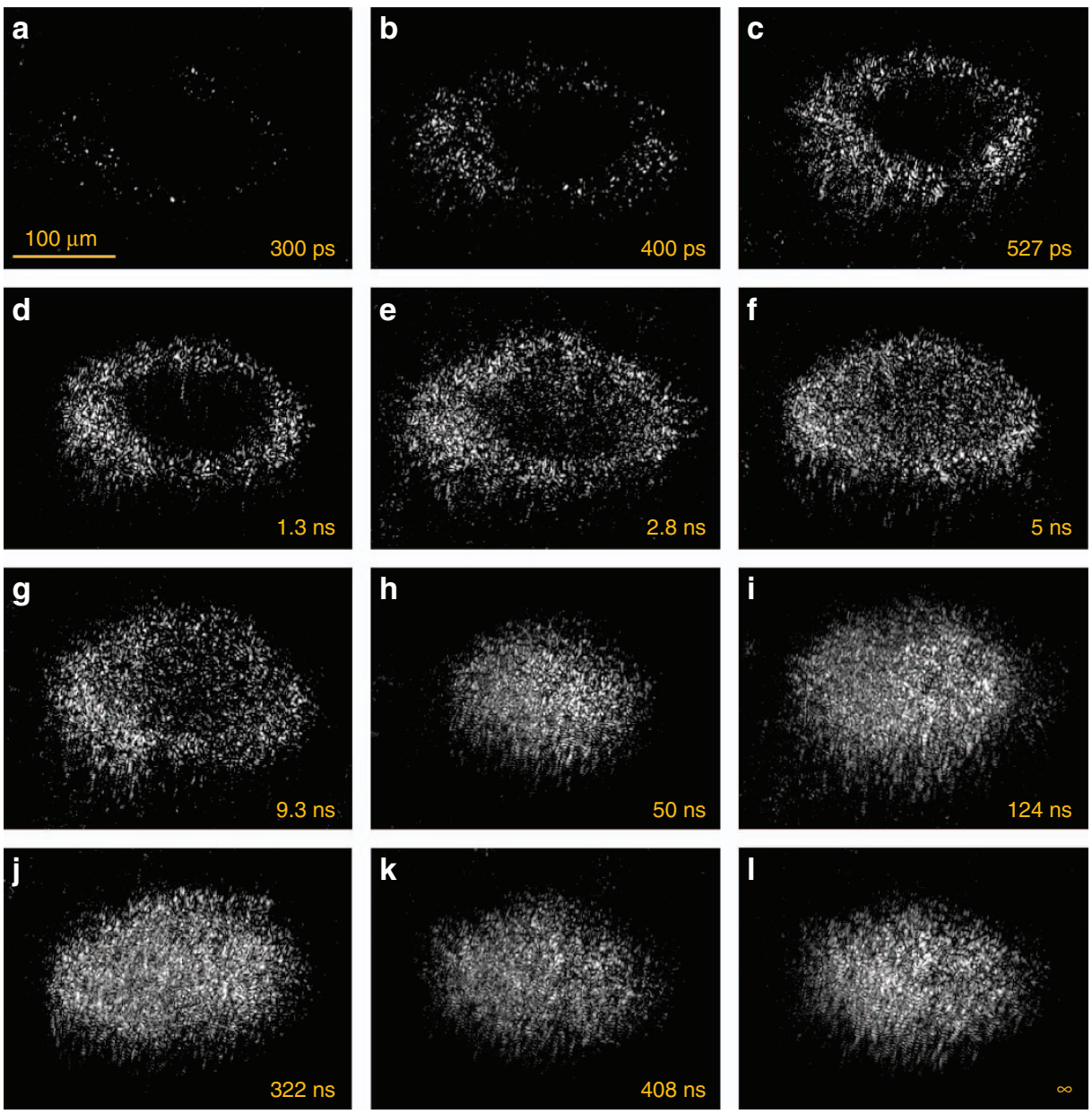

Figure $4(\mathrm{a}-\mathrm{I})$ CCD images of a $\mathrm{Zn}$ surface at various delay times following a pump pulse at a fluence of $1.0 \mathrm{~J} \mathrm{~cm}^{-2}$.

center also change from being mostly nanostructures to being mostly microstructures. At this and higher laser fluences, nanostructures can still be produced at the edge of the ablated spot because of the Gaussian profile of the laser beam. Therefore, the spatial evolution of the ring surface pattern observed between $300 \mathrm{ps}$ and $2.8 \mathrm{~ns}$ in Figure 4 is attributed to the fact that nanostructures first form at the periphery and the center region is later populated with microstructures; as shown earlier with regard to the timescale of morphological fluctuations $\left(t_{\mathrm{p}} \approx L / v_{\mathrm{sl}}\right)$, the time required to form nanostructures (smaller $L$ ) is shorter than that required to form microstructures (larger $L$ ). Another possible explanation for the central dark spot is the formation of highly light-absorptive matter in the central part of the interaction area, possibly due to the formation of an ablation plasma that masks the surface structures. Over time, such an ablation plasma would become more transparent, and the surface structures in the central part of the ablated area would become more visible. We note that the absorption of laser light by an ablation plasma has been previously observed in double-pulse ablation studies $^{28,29}$

Next, we study the mechanisms of the evolution of the transient surface structures from their first appearance $\left(t_{1}\right)$ up through the onset of solidification $\left(t_{2}\right)$. Several possible ablation mechanisms exist $^{25,26,30-32}$, that is, evaporation ${ }^{31}$, thermomechanical fragmentation/spallation ${ }^{25,26}$ and phase explosion ${ }^{25,32}$. To identify the ablation mechanisms that are responsible to our observations, we estimate the maximum lattice temperature $T_{\mathrm{m}}$ (Refs. 21,33):

$$
T_{\mathrm{m}} \approx T_{0}+\frac{F_{\mathrm{A}}}{c_{i}} \frac{\beta \alpha}{\beta+\alpha}, \beta=\sqrt{\frac{g}{k}}
$$

where $T_{0}$ is room temperature, $c_{i}$ is the specific heat of the lattice, $\alpha$ is the light attenuation coefficient, $g$ is the electron-phonon coupling factor, $k$ is the thermal conductivity, and $F_{\mathrm{A}}=(1-R) F$ is the absorbed laser fluence, where $R$ is the reflectance of $\mathrm{Zn}$ at the wavelength of the laser. The reflectance of a metal surface depends on its condition after polishing, the angle of the incident light and the laser fluence ${ }^{20}$. The key to obtaining a reliable estimate of the surface temperature is to accurately determine the value of the sample reflectance under our experimental conditions. To do so, we perform a rigorous surface reflectance measurement using a hemiellipsoidal collector to collect both the specular and diffuse reflected light, as first demonstrated in Ref. 20. The measured reflectance in a fluence range of $0.01-1.0 \mathrm{~J} \mathrm{~cm}^{-2}$ is $R=0.55-0.57$, as shown in Table 1 . Table 1 also shows the surface temperatures estimated using $T_{0}=300 \mathrm{~K}$, $c_{\mathrm{i}}=2.78 \times 10^{6} \mathrm{~J} \mathrm{~m}^{-3} \mathrm{~K}^{-1}$ (Ref. 34), $\alpha=7.5 \times 10^{7} \mathrm{~m}^{-1}$ (Ref. 34), $g=6 \times 10^{16} \mathrm{~W} \mathrm{~m}^{-3} \mathrm{~K}^{-1}$ (Ref. 34), and $k=116 \mathrm{~W} \mathrm{~m}^{-1} \mathrm{~K}^{-1}$. As we can see, at the damage threshold laser fluence of $0.022 \mathrm{~J} \mathrm{~cm}^{-2}, T_{\mathrm{m}}$ is estimated to be $890 \mathrm{~K}$. This value is only slightly higher than the melting point of $\mathrm{Zn}(693 \mathrm{~K})$, indicating that Equation (2) provides a very good estimate of the surface temperature. At $F=0.1 \mathrm{~J} \mathrm{~cm}^{-2}$, the surface temperature is estimated to be $2970 \mathrm{~K}$, exceeding the boiling point of zinc $(1180 \mathrm{~K})$, and this laser fluence is clearly in the ablation 

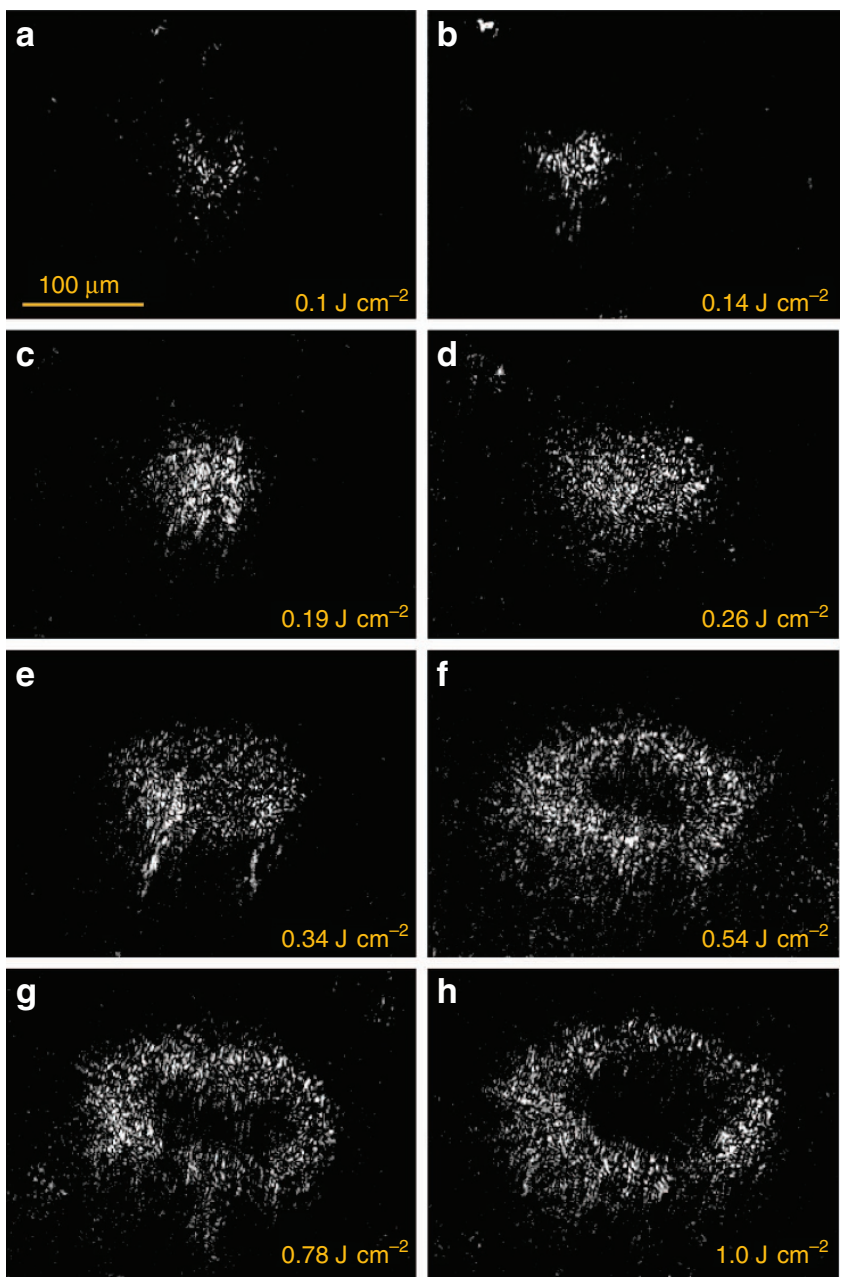

Figure 5 (a-h) CCD images of Zn surfaces at a fixed delay time of 527 ps following pump pulses at various laser fluence values.

regime. At laser fluences of $0.14 \mathrm{~J} \mathrm{~cm}^{-2}$ or above, the estimated surface temperatures are above the thermodynamic critical point $T_{\mathrm{c}}$ $(3190 \mathrm{~K})$, suggesting that the laser-excited material becomes a supercritical fluid (with no distinction between the liquid and vapor phases). The surface temperature for ablation at $F=0.1 \mathrm{~J} \mathrm{~cm}^{-2}$ can lead to either vaporization ${ }^{31}$ or thermomechanical fragmentation/ spallation $^{25,26}$. However, it is believed that the predominant contribution to ablation is from thermomechanical fragmentation/spallation, whereas the role of vaporization is not essential ${ }^{35}$. Therefore, we believe that our results at a fluence of $0.1 \mathrm{~J} \mathrm{~cm}^{-2}$ are mostly related to ablation through the spallation mechanism. In the spallation regime, an ultrashort laser pulse induces fast isochoric heating of the surface layer, which causes a buildup of high stress, cavitation, nanofoam formation and thermomechanical spallation (ablation) of a liquid layer on the surface due to the relaxation of tensile stresses developed in the surface layer $25,26,36-38$. For a laser fluence of higher than $0.14 \mathrm{~J} \mathrm{~cm}^{-2}$, the surface temperature is estimated to be significantly higher than $0.9 T_{c}$, which is the characteristic threshold temperature for phase explosion $^{32}$. Phase explosion occurs at laser fluences below those for substantial ionization and plasma formation ${ }^{38}$. It is known that plasma formation causes a significant decrease in reflectance ${ }^{20,39}$. Our reflectance measurements show that a significant decrease in the reflectance of the $\mathrm{Zn}$ surface occurs at laser fluences above $1.1 \mathrm{~J} \mathrm{~cm}^{-2}$; see Table 1. This indicates that in the laser fluence range of
$0.14-1.1 \mathrm{~J} \mathrm{~cm}^{-2}$, the ablation is primarily caused by phase explosion. Therefore, the transient structural dynamics between $t_{1}$ and $t_{2}$ originate from either the spallation mechanism or the phase explosion mechanism, depending on the laser fluence. As shown in Figure 3, both $t_{1}$ and $t_{2}$ depend on the laser fluence. The start time of solidification, $t_{2}$, increases with laser fluence, indicating that a longer time is required for solidification to occur at a higher laser fluence.

Finally, we study the mechanism of complete solidification and its associated time, $t_{3}$. After ablation, rapid cooling due to high electronic heat conduction causes solidification of the melted surface layer, giving rise to permanent surface structures. The cooling and resolidification of the melted surface layer in the spallation regime has previously been modeled using the atomistic molecular dynamics method combined with a continuum-level two-temperature model ${ }^{36,37}$. In these works, the time of complete resolidification was predicted to be $2.5 \mathrm{~ns}$ for $\mathrm{Al}$ (Ref. 36) and 1.4-1.7 ns for Ag (Ref. 37). These timescales are approximately two orders of magnitude smaller than our observed complete solidification time, $t_{3}$, for $\mathrm{Zn}$ (Table 1 ). It is generally believed that the cooling of an ultrafast laser-heated metal surface is dominated by thermal conduction ${ }^{21,33}$. Under these conditions, the cooling time $t_{\text {cool }}$ is estimated to $\mathrm{be}^{21}$

$$
t_{\mathrm{cool}} \approx \delta^{2} / 4 \chi, \quad \delta=\left(\frac{18 I_{\mathrm{A}} k}{g^{2} T_{0}}\right)^{1 / 3}
$$

where $\chi$ is the thermal diffusivity and $I_{\mathrm{A}}$ is the absorbed laser intensity. The estimated values of $t_{\text {cool }}$ for $\mathrm{Zn}$ are shown in Table 1, where we see that $t_{\text {cool }}$ is significantly shorter than the observed $t_{3}$. Thus, both the model predictions ${ }^{36,37}$ and the estimation based on Equation (3) predict significantly faster cooling than that observed here in our experiments, indicating that the current understanding of the rapid cooling process is incomplete. To explain this discrepancy, we speculate that the slower cooling process is due to an enhanced thermal coupling that has previously been observed and modeled ${ }^{40,41}$, where it was found that a significant amount of thermal energy remains in a metal following fs laser ablation because of the transfer of thermal energy from the ablation plume back to the sample on a timescale that is much longer than the laser pulse. Our speculation is supported by previous studies of residual thermal coupling in the ablation of $\mathrm{Zn}$ (Ref. 40), which indicates that the onset of the enhanced thermal coupling occurs at $F=0.45 \mathrm{~J} \mathrm{~cm}^{-2}$ and is correlated with an abrupt increase in the start time of solidification, $t_{2}$, above $0.54 \mathrm{~J} \mathrm{~cm}^{-2}$, as seen in Figure 3. Furthermore, it has been shown that the heating of a sample through the transfer of energy from the ablation plasma causes ultradeep drilling of the material ${ }^{42}$. In addition to the enhanced thermal coupling, surface cooling can also be slowed by the latent heat of resolidification and by exothermic chemical reactions induced during laser ablation ${ }^{43}$. In one example of the large contribution of chemical sources of energy, this contribution has been shown to reach up to $30 \%$ of the incident laser energy during the nanosecond laser ablation of zinc in $\operatorname{air}^{43}$.

\section{CONCLUSIONS}

In conclusion, for the first time, we have captured and visualized the complete temporal and spatial evolution of the fs laser-induced surface structural dynamics of metals by utilizing a time-resolved optical imaging technique. We find that transient surface structures first appear at a delay time on the order of $100 \mathrm{ps}$, and this is attributed to ablation driven by pressure relaxation in the surface layer. At lower laser fluences that favor nanostructure formation, the transient surface structures first appear in the central portion of the ablated spot. 
At higher laser fluences that favor microstructure formation, nanostructures first emerge at the periphery, and the center region is later populated with microstructures. For zinc, the predicted cooling time is two orders of magnitude shorter than the time found in our observations, and we speculate that this slower cooling process is due to an enhanced thermal coupling phenomenon that has been previously observed. The visualization and control of surface structural dynamics not only are of fundamental importance for understanding the fs laser-induced responses of materials but also pave the way for the design of new material functionalities through surface structuring.

\section{CONFLICT OF INTEREST}

The authors declare no conflict of interest.

\section{ACKNOWLEDGEMENTS}

This work was supported by the US Army Research Office, the Bill \& Melinda Gates Foundation, and the National Natural Science Foundation of China.

1 Gamaly EG. Femtosecond Laser-Matter Interaction: Theory, Experiments and Applications. Singapore: Pan Stanford Publishing. 2011.

2 Vorobyev AY, Guo C. Direct femtosecond laser surface nano/microstructuring and its applications. Laser Photon Rev 2013; 7: 385-407.

3 Ahmmed KMT, Grambow C, Kietzig AM. Fabrication of micro/nano structures on metals by femtosecond laser micromachining. Micromachines 2014; 5: 1219-1253.

4 Cheng J, Liu CS, Shang S, Liu D, Perrie W et al. A review of ultrafast laser materials micromachining. Opt Laser Technol 2013; 46: 88-102.

5 Li L, Hong MH, Schmidt M, Zhong ML, Malshe A et al. Laser nano-manufacturingstate of the art and challenges. CIRP Ann-Manufact Technol 2011; 60: 735-755.

6 Siders CW, Cavalleri A, Sokolowski-Tinten K, Tóth C, Guo T et al. Detection of nonthermal melting by ultrafast X-ray diffraction. Science 1999; 286: 1340-1342.

7 Rousse A, Rischel C, Fourmaux S, Uschmann I, Sebban S et al. Non-thermal melting in semiconductors measured at femtosecond resolution. Nature 2001; 410: 65-68.

8 Siwick BJ, Dwyer JR, Jordan RE, Miller RJD. An atomic-level view of melting using femtosecond electron diffraction. Science 2003; 302: 1382-1385.

9 Sokolowski-Tinten K, Barty A, Boutet S, Shymanovich U, Chapman H et al. Short-pulse laser induced transient structure formation and ablation studied with time-resolved coherent XUV-scattering. AIP Conf Proc 2010; 1278: 373-379.

10 Downer MC, Fork RL, Shank CV. Femtosecond imaging of melting and evaporation at a photoexcited silicon surface. J Opt Soc Am B 1985; 2: 595-599.

11 Bonse J, Bachelier G, Siegel J, Solis J, Sturm H. Time- and space-resolved dynamics of ablation and optical breakdown induced by femtosecond laser pulses in indium phosphide. J Appl Phys 2008; 103: 054910.

12 von der Linde D, Sokolowski-Tinten K. The physical mechanisms of short-pulse laser ablation. Appl Surf Sci 2000; 154-155: 1-10.

13 Sokolowski-Tinten K, Bialkowski J, Cavalleri A, von der Linde D, Oparin A et al. Transient states of matter during short pulse laser ablation. Phys Rev Lett 1998; 81: 224-227.

14 Bonse J, Bachelier G, Siegel J, Solis J. Time- and space-resolved dynamics of melting, ablation, and solidification phenomena induced by femtosecond laser pulses in germanium. Phys Rev B 2006; 74: 134106.

15 Domke M, Rapp S, Schmidt M, Huber HP. Ultrafast pump-probe microscopy with high temporal dynamic range. Opt Express 2012; 20: 10330-10338.

16 Hernandez-Rueda J, Puerto D, Siegel J, Galvan-Sosa M, Solis J. Plasma dynamics and structural modifications induced by femtosecond laser pulses in quartz. Appl Surf Sci 2012; 258: 9389-9393.

17 Barty A, Boutet S, Bogan MJ, Hau-Riege S, Marchesini S et al. Ultrafast single-shot diffraction imaging of nanoscale dynamics. Nat Photonics 2008; 2: 415-419.

18 Boustany NN, Boppart SA, Backman V. Microscopic imaging and spectroscopy with scattered light. Annu Rev Biomed Eng 2010; 12: 285-314.

19 Vorobyev AY, Guo C. Femtosecond laser nanostructuring of metals. Opt Express 2006; 14: 2164-2169.
20 Vorobyev AY, Guo C. Reflection of femtosecond laser light in multipulse ablation of metals. J Appl Phys 2011; 110: 043102.

21 Agranat MB, Ashitkov SI, Fortov VE, Kirillin AV, Kostanovskii AV et al. Use of optical anisotropy for study of ultrafast phase transformations at solid surfaces. Appl Phys $A$ 1999; 69: 637-640.

22 Bonse J, Wiggins SM, Solis J. Phase transitions induced by femtosecond laser pulse irradiation of indium phosphide. App/ Surf Sci 2005; 248: 151-156.

23 Assael MJ, Armyra IJ, Brillo J, Stankus SV, Wu JT et al. Reference data for the density and viscosity of liquid cadmium, cobalt, gallium, indium, mercury, silicon, thallium, and zinc. J Phys Chem Ref Data 2012; 41: 033101.

24 Nogi K, Ogino K, McLean A, Miller WA. The temperature coefficient of the surface tension of pure liquid metals. Metall Trans B 1986; 17: 163-170.

25 Leveugle E, Ivanov DS, Zhigilei LV. Photomechanical spallation of molecular and metal targets: molecular dynamics study. Appl Phys A 2004; 79: 1643-1655.

26 Inogamov NA, Faenov AY, Zhakhovskii VV, Skobelev IY, Khokhlov VA et al. Interaction of short laser pulses in wavelength range from infrared to X-ray with metals, semiconductors, and dielectrics. Contrib Plasma Phys 2011; 51: 361-366.

27 Lide DR. CRC Handbook of Chemistry and Physics 84th edn. Boca Raton: CRC Press. 2003.

28 Semerok A, Dutouquet C. Ultrashort double pulse laser ablation of metals. Thin Solid Films 2004; 453-454: 501-505.

29 Povarnitsyn ME, Itina TE, Khishchenko KV, Levashov PR. Suppression of ablation in femtosecond double-pulse experiments. Phys Rev Lett 2009; 103: 195002.

30 Bulgakova NM, Stoian R, Rosenfeld A, Hertel IV. Continuum models of ultrashort pulsed laser ablation. In: Miotello A, Ossi P (eds). Laser-Surface Interactions for New Materials Production. Berlin: Springer, 2010; 130: 81-97.

31 Anisimov SI, Luk'yanchuk BS. Selected problems of laser ablation theory. Phys Uspekhi 2002; 45: 293-324.

32 Miotello A, Kelly R. Critical assessment of thermal models for laser sputtering at high fluences. Appl Phys Lett 1995; 67: 3535-3537.

33 Anisimov SI, Rethfeld B. On the theory of ultrashort laser pulse interaction with a metal. Proc SPIE 1997; 3093: 192-203.

34 Shugaev MV, Bulgakova NM. Thermodynamic and stress analysis of laser-induced forward transfer of metals. Appl Phys A 2010; 101: 103-109.

35 Inogamov NA, Zhakhovskii WV, Khokhlov VA. Jet formation in spallation of metal film from substrate under action of femtosecond laser pulse. J Exp Theor Phys 2015; 120: 15-48.

36 Inogamov NA, Zhakhovsky VV, Khokhlov VA, Ashitkov SI, Emirov YN et al. Ultrafast lasers and solids in highly excited states: results of hydrodynamics and molecular dynamics simulations. J Phys Conf Ser 2014; 510: 012041.

37 Wu CP, Christensen MS, Savolainen JM, Balling P, Zhigilei LV. Generation of subsurface voids and a nanocrystalline surface layer in femtosecond laser irradiation of a single-crystal Ag target. Phys Rev B 2015; 91: 035413.

38 Zhigilei LV, Lin ZB, Ivanov DS. Atomistic modeling of short pulse laser ablation of metals: connections between melting, spallation, and phase explosion. J Phys Chem C 2009; 113: 11892-11906.

39 Kirkwood SE, Tsui YY, Fedosejevs R, Brantov AV, Bychenkov VY. Experimental and theoretical study of absorption of femtosecond laser pulses in interaction with solid copper targets. Phys Rev B 2009; 79: 144120

40 Vorobyev AY, Guo C. Enhanced energy coupling in femtosecond laser-metal interactions at high intensities. Opt Express 2006; 14: 13113-13119.

41 Bulgakova NM, Zhukov VP, Vorobyev AY, Guo C. Modeling of residual thermal effect in femtosecond laser ablation of metals: role of a gas environment. Appl Phys A 2008; 92: 883-889.

42 Bulgakova NM, Evtushenko AB, Shukhov YG, Kudryashov SI, Bulgakov AV. Role of laser-induced plasma in ultradeep drilling of materials by nanosecond laser pulses. Appl Surf Sci 2011; 257: 10876-10882.

43 Vorob'ev AY, Dorofeev IA, Libenson MN. Concerning the chemical source of energy in a laser flare. Sov Tech Phys Lett 1992; 18: 172-173.

(c) (1) This work is licensed under a Creative Commons AttributionBY NG SA NonCommercial-ShareAlike 4.0 International License. The images or other third party material in this article are included in the article's Creative Commons license, unless indicated otherwise in the credit line; if the material is not included under the Creative Commons license, users will need to obtain permission from the license holder to reproduce the material. To view a copy of this license, visit http:// creativecommons.org/licenses/by-nc-sa/4.0/

(C) The Author(s) 2017 